\title{
On High Precision Methods for Computing Integrals Involving Bessel Functions
}

\author{
By Bruno Gabutti
}

Abstract. The technique of Bakhvalov and Vasil'eva for evaluating Fourier integrals is generalized to integrals involving exponential and Bessel functions.

1. Introduction. In some problems of high energy nuclear physics (see Glauber [5]) one has to evaluate integrals of the form

$$
I(\omega)=\int_{0}^{\infty} e^{-x^{2}} J_{0}(\omega x) f\left(x^{2}\right) x d x,
$$

where $\omega$ is a positive parameter.

For large values of $\omega$ it is straightforward to verify that the integrand function is strongly oscillating; standard methods (for instance Gauss-Laguerre quadrature) and a nonstandard method (see Steen, Byrne and Gelbard [12]) which do not take this into account are inadequate.

Subdividing the interval (1) into subintervals where ends are zeros of $J_{0}(\omega x)$ results in having to sum a large number of terms having alternate signs. Even when Longman's technique (see [8]) is used we found it impossible to obtain high accuracy.

Such a situation is very similar to the one that we meet in the calculation of integrals of the form

$$
\int_{a}^{b} g(x) e^{i \omega x} d x
$$

Linz [7] suggests a method which, using the Abel transform, reduces the calculation of (1) to a sequence of calculation of integrals of type (2) with $g(x)=e^{-x^{2}} f(x) x$.

In recent years many efficient methods for the computation of (2) have been developed; we mention Bakhvalov and Vasil'eva [1], Lyness [9], Piessens and Haegemans [11], Patterson [10].

In this paper we suggest a method which is an adaptation of the Bakhvalov and Vasil'eva method to the integral (1). It will appear that our formulas are more manageable than the one we would have obtained by arranging the integral (1) in the form (2).

2. The Method. We consider the expansion in Laguerre polynomials of the function $f\left(x^{2}\right)$ in the interval $[0, \infty)$

$$
f\left(x^{2}\right) \simeq \sum_{k=0}^{N} b_{k} L_{k}\left(x^{2}\right)
$$

Received March 23, 1978 ; revised September 19, 1978.

AMS (MOS) subject classifications (1970). Primary 65D30; Secondary 35A40. 
with

$$
b_{k}=\int_{0}^{\infty} e^{-y} L_{k}(y) f(y) d y .
$$

Since (see Gradshteyn and Ryzhik [6, 7.421.2])

$$
\int_{0}^{\infty} e^{-x^{2}} J_{0}(\omega x) L_{k}\left(x^{2}\right) x d x=\frac{e^{-\omega^{2} / 4}}{2 k !}\left(\frac{\omega}{2}\right)^{2 k}
$$

we can express $I(\omega)$ in the form

$$
I(\omega) \simeq \sum_{k=0}^{N} b_{k} V_{k}
$$

where

$$
V_{k}=\frac{e^{-\omega^{2} / 4}}{2 k !}\left(\frac{\omega}{2}\right)^{2 k}
$$

Formula (6) requires the coefficients $b_{k}$. In some easy cases analytic expressions for $b_{k}$ are known.

In general, one has to approximate $b_{k}$ by $b_{k}^{(N+1)}$, the approximation to (4) obtained using the $(N+1)$-point Gauss-Laguerre quadrature formula: we define an approximation to $I(\omega)$ by

$$
I^{(N)}(\omega)=\sum_{k=0}^{N} b_{k}^{(N+1)} V_{k}
$$

where $I^{N}(\omega)$ is an approximation value of $I(\omega)$.

In cases in which one requires the value of $I(\omega)$ for many different values of $\omega$, the same values of $b_{k}^{(N)}$ may be used in each calculation.

For the coefficients $V_{k}$ it is convenient to utilize the simple recurrence formulas

$$
\begin{gathered}
V_{k-1}=\frac{4 k}{\omega^{2}} V_{k} ; \quad k=0,1, \ldots, k_{\omega}, \\
V_{k+1}=\frac{\omega^{2}}{4 k} V_{k} ; \quad k=k_{\omega}+1, k_{\omega}+2, \ldots, N,
\end{gathered}
$$

with

$$
k_{\omega}=\left[\frac{\omega^{2}}{4}\right]
$$

([x] denotes the integer part of $x$ ). $V_{k_{\omega}}$, the starting value for (9)-(10), may be calculated using Stirling's formula in (7). This gives an estimate

$$
V_{k_{\omega}} \simeq \frac{1}{(2 \pi)^{1 / 2} \omega},
$$

which can be improved simply by using a larger number of terms in asymptotic expansion of $k$ !; for example

$$
V_{k_{\omega}}=\frac{1}{(2 \pi)^{1 / 2}}\left[\omega\left(1+\frac{1}{3 \omega^{2}}+\frac{1}{18 \omega^{4}}-\frac{139}{810 \omega^{6}}-\frac{571}{9720 \omega^{8}}+R\right)\right]^{-1}
$$

where

$$
|R| \leqslant \omega^{-10}
$$


Therefore, the values of $V_{k}$ are affected by the propagation of the truncation error inherent at (13). Such an error (which can be reduced by using more terms in the asymptotic expansion of $k$ !) can be easily controlled. When $\omega$ is so small that the requested accuracy is not attained $(\omega \leqslant 12$ in our numerical experience) it is convenient to use (9) with

$$
V_{0}=1 / 2 e^{-\omega^{2}} \text {. }
$$

When $\omega<<1$ the drawbacks described in the introduction are tolerable and the integral can be immediately calculated using any standard method.

3. Error Analysis. Formula (6) supplies the same approximation of the integral (1) given by $(N+1)$-point interpolatory Gaussian quadrature formulas; both (6) and (8) are exact whenever $f(y)$ is a polynomial of degree less than or equal to $N$. The approximation error in (6) will be, therefore, formally given by

$$
E_{N+1}=(-1)^{N+1} \int_{0}^{\infty} e^{-x^{2}} J_{0}(\omega x) f^{(N+1)}(\zeta(x)) L_{N+1}(x) x d x,
$$

where $\zeta(x)$ is an unknown function and is bounded by

$$
\left|E_{N+1}\right| \leqslant M_{N+1} \int_{0}^{\infty}\left|e^{-x^{2}} J_{0}(\omega x) x L_{N+1}(x)\right| d x,
$$

where $M_{N+1}$ is the max of absolute value of the $(N+1)$ th derivative of $f(y)$ in $[0, \infty)$.

Bound (17) is theoretically important because it allows us to state that $\left|E_{N+1}\right|$ is bounded when the $(N+1)$ th derivative (if it exists) of $f(y)$ is bounded but, for practical purposes, is unlikely to be useful.

When $f(y)$ is an analytic function a more detailed analysis of the error can be carried out using the residue method suggested by Barrett [3] .

In practice, when $f(y)$ is any function known only at a number of discrete points or in terms of a complicated analytic expression, we adopt a convergence test for (6) of the type suggested by Clenshaw and Curtis [4]; this requires

$$
h^{2}\left|\frac{b_{k} V_{k}}{I^{(k)}(\omega)}\right|, h\left|\frac{b_{k+1} V_{k+1}}{I^{(k+1)}(\omega)}\right|,\left|\frac{b_{k+2} V_{k+2}}{I^{(k+2)}(\omega)}\right| \leqslant E P S ; \quad k=k_{\omega}, k_{\omega}+1, \ldots,
$$

where $E P S$ is the requested precision and $0<h<1$ is a coefficient which is determined empirically. (In our numerical experiments we found values of $h$ satisfying $1 / 8<h<1 / 9$ to be adequate.)

As far as the error $\left(b_{k}-b_{k}^{(N+1)}\right)$ is concerned there exists a bound of the same form (17). The case in which $f(y)$ is known only at a number of discrete values of $y$, the convergence of $b_{k}^{(N+1)}$ to $b_{k}$ has been analyzed by Balàzs and Turan [2] .

In practice we calculate approximation to $b_{k}$ using standard techniques, namely, subdividing the interval in the following way:

$$
\begin{aligned}
b_{k}= & \int_{0}^{\infty} e^{-y} L_{k}(y) f(y) d y=\int_{0}^{a} e^{-y} L_{k}(y) f(y) d y \\
& +e^{-a} \int_{a}^{\infty} e^{-y} L_{k}(y+a) f(y+a) d y=c_{k}+d_{k} .
\end{aligned}
$$


When $a$ is sufficiently large, then $d_{k}$ is negligible with respect to $c_{k}$ and $c_{k}$ can be calculated using the Gauss-Legendre formula in $(0, a)$. Further subdivision of the same nature may be used if necessary.

4. Scaling to Enhance Convergence. The effect of rescaling the whole problem can be computationally advantageous. Let

$$
x=\alpha t,
$$

where $\alpha$ is a scaling factor; the integral (1) may be written in the form

$$
I_{\alpha}(\omega)=\alpha^{2} \int_{0}^{\infty} e^{-t^{2}} J_{0}(\omega \alpha t) g\left(\alpha^{2} t^{2}\right) t d t
$$

where

$$
g\left(\alpha^{2} t^{2}\right)=e^{\left(1-\alpha^{2}\right) t^{2}} f\left(\alpha^{2} t^{2}\right)
$$

Using for (19), the procedure of Section 2 we see that for fixed $\omega$ the rate of convergence of summation (6) depends also on $\alpha$ as the following example illustrates:

$$
I(\omega)=\int_{0}^{\infty} e^{-x^{2}} J_{0}(\omega x) x \sin x^{2} d x .
$$

In Table 1 the number of terms $N$ required to obtain at least 12 correct significant figures for (20) with $\omega=6$ are listed for several values of $\alpha$. All calculations were performed in double precision arithmetic on an IBM 360/75 computer (about 15 significant decimal digits).

TABLE 1

\begin{tabular}{ccccccccc}
\hline$\alpha$ & 0.33 & 0.47 & 0.57 & 0.67 & 0.82 & 1.0 & 1.2 & 1.5 \\
\hline$N$ & 37 & 34 & 32 & 31 & 31 & 34 & 41 & 54 \\
\hline
\end{tabular}

We observe that the optimum value of $\alpha(\alpha \simeq 0.7)$ is approximately the one for which the Laguerre expansion of $e^{\left(1-\alpha^{2}\right) t^{2}} \sin \left(\alpha^{2} t^{2}\right)$ becomes divergent. Unfortunately, we do not know how to estimate this optimum value a priori. One empirical but expensive way is to compare values of $I_{\alpha}(\omega)$ for various values of $\alpha$.

5. Scaling to Avoid Loss of Numerical Significance. The method outlined in Section 2 proved to be efficient. From (9)-(10) we see that the terms $V_{k}, k=0$, $1, \ldots$, are increasing for $0 \leqslant k \leqslant k_{\omega}$ and decreasing for $k>k_{\omega}$; and, in practice, the behavior of the coefficients $\left|b_{k} V_{k}\right|$ usually follows the same pattern. Therefore, the number of terms $N$ required in (6) increases monotonically with $\omega$; for $k>k_{\omega}$, the summation (6) is quickly convergent.

In spite of this, in some cases the computation of (6) must be carried out carefully. This is because the size of terms $b_{k} V_{k}$ at first increases and then decreases (like, for example, the size of individual terms in the convergent series for $\sin x$ when $x=$ 10). Thus, using finite length arithmetic, the attainable accuracy is limited to $2^{-m}\left|b_{M} V_{M}\right|$, where $\left|b_{M} V_{M}\right|$ is the largest term in the series and $2^{-m}$ is the machine 
accuracy parameter. (On machines using base 2 arithmetic, this is the word length of the mantissa in floating-point representation of a number in the machine.)

Such a situation is illustrated by example (20): when $\omega=20$, the true value of $I(\omega)$ is $0.59 \cdot 10^{-22}$. We find the first term $b_{0} V_{0} \simeq 10^{-43}$. The terms increase in magnitude, the largest being $\left|b_{M} V_{M}\right|=0.32 \cdot 10^{-14}$ with $M=80$. Thereafter, the size of the terms decrease.

Although one can go on calculating individual terms, one knows at this stage that there is no point in going beyond the stage at which $\left|b_{M^{\prime}} V_{M^{\prime}}\right| \simeq 10^{-15} \cdot 0.32 \cdot$ $10^{-14}=0.32 \cdot 10^{-29}$ and that when this is done the relative accuracy cannot exceed $10^{-7}$. In fact, stopping the summation (8) after $N$ terms so that the convergence test (18) with EPS $=10^{-12}, h=1 / 8$ is attained, the term $b_{N} V_{N}$ with $N=165$ is of size $10^{-34}$; but this information is of no use. These numbers are listed in the first line of Table 2 .

The situation illustrated in this example may be improved by introducing a suitable scaling parameter $\alpha$. Now we consider $\alpha$ using a criterion different than the one used in Section 4; instead of choosing $\alpha$ to enhance the convergence rate, one chooses $\alpha$ sufficiently large to hold $\left|b_{M} V_{M}\right|$ less than $2^{m} \operatorname{EPS}|I(\omega)|$. The required accuracy for example (20) with $\omega=20$ is obtained as we see in Table 2 .

TABLE 2

\begin{tabular}{|r|c|r|r|r|r|r|r|r|}
\hline$\alpha$ & $\left|b_{0} V_{0}\right|$ & $M$ & $\left|b_{M} V_{M}\right|$ & $M^{\prime}$ & $\left|b_{M^{\prime}} V_{M^{\prime}}\right|$ & $N$ & $\left|b_{N} V_{N}\right|$ & $\begin{array}{l}\text { Relative } \\
\text { accuracy } \\
\text { of numer- } \\
\text { ical results }\end{array}$ \\
\hline 1.0 & $0.93 \cdot 10^{-44}$ & 72 & $0.32 \cdot 10^{-14}$ & 152 & $0.28 \cdot 10^{-29}$ & 165 & $0.58 \cdot 10^{-34}$ & $0.7 \cdot 10^{-7}$ \\
1.2 & $0.50 \cdot 10^{-63}$ & 109 & $0.46 \cdot 10^{-18}$ & 200 & $0.37 \cdot 10^{-32}$ & 207 & $0.30 \cdot 10^{-34}$ & $0.9 \cdot 10^{-11}$ \\
1.4 & $<10^{-78}$ & 151 & $0.44 \cdot 10^{-20}$ & $>266$ & $<0.24 \cdot 10^{-34}$ & 266 & $0.24 \cdot 10^{-34}$ & $0.3 \cdot 10^{-12}$ \\
1.6 & $<10^{-78}$ & 209 & $0.28 \cdot 10^{-21}$ & $>333$ & $<0.17 \cdot 10^{-34}$ & 333 & $0.17 \cdot 10^{-34}$ & $0.1 \cdot 10^{-11}$ \\
\hline
\end{tabular}

$b_{M} V_{M}$ is the largest term in series.

$b_{M^{\prime}} V_{M^{\prime}}$ is the first term less than $10^{-15} b_{M} V_{M}$.

$b_{N} V_{N}$ is the first term which satisfies criterion (18) with $E P S=10^{-12}, h=1 / 8$.

6. Some Numerical Examples. Up to this point, the same example has been used simply to facilitate the detailed description of the scaling procedure. While the full generality of the technique is not known, we have used it with varying success in the following problems:

Example 1. We consider two simple cases for which formula (6) is exact with a finite number $N$ in terms

$$
\begin{aligned}
& f\left(x^{2}\right)=1, \\
& f\left(x^{2}\right)=x^{12} .
\end{aligned}
$$

Here we have found scaling neither necessary nor useful. This is clearly evidenced in Table 3 where, assuming $\omega=4$, the number of terms $N$ required to satisfy the 
TABLE 3

\begin{tabular}{|c|c|c|c|c|}
\hline & \multicolumn{2}{|c|}{ (Example (23) } & \multicolumn{2}{c|}{ Example (24) } \\
\hline$\alpha$ & $N$ & $\begin{array}{l}\text { Relative accu- } \\
\text { racy of numer- } \\
\text { ical results }\end{array}$ & $N$ & $\begin{array}{l}\text { Relative accu- } \\
\text { racy of numer- } \\
\text { ical results }\end{array}$ \\
\hline 0.50 & 26 & $0.1 \cdot 10^{-13}$ & 33 & $0.6 \cdot 10^{-13}$ \\
0.71 & 22 & $0.2 \cdot 10^{-14}$ & 29 & $0.1 \cdot 10^{-11}$ \\
0.87 & 17 & $0.4 \cdot 10^{-15}$ & 23 & $0.2 \cdot 10^{-11}$ \\
1.00 & $3 *$ & 0. & $9 *$ & $0.4 \cdot 10^{-15}$ \\
1.12 & 16 & $0.1 \cdot 10^{-14}$ & 22 & $0.2 \cdot 10^{-11}$ \\
1.32 & 24 & $0.3 \cdot 10^{-14}$ & 29 & $0.3 \cdot 10^{-11}$ \\
1.50 & 29 & $0.3 \cdot 10^{-13}$ & 34 & $0.6 \cdot 10^{-12}$ \\
\hline
\end{tabular}

(*) The exact result is of course obtained with $N=1$ and $N=7$, respectively. However, two further iterations were required to satisfy convergence criterion (18) in each case.

convergence test (18) with $E P S=10^{-12}, h=1 / 8$ and the relative accuracy of the result obtained are listed for several values of $\alpha$.

Example 2. We consider four different behaviors of $f\left(x^{2}\right)$ for $x \rightarrow \infty$, namely: exponential decreasing, exponential increasing, rational decreasing, rational increasing. Let $\omega=6$ and

$$
\begin{gathered}
f\left(x^{2}\right)=e^{-x^{2} / 2} x^{8}-1, \\
f\left(x^{2}\right)=e^{x^{2} / 4} x^{6}, \\
f\left(x^{2}\right)=x^{-2 / 5}, \\
f\left(x^{2}\right)=x^{28 / 5} .
\end{gathered}
$$

In all these cases the method has been effective and the scaling procedure has been useful in accelerating the convergence of (8) (as in the example in Section 4), but the accuracy of numerical results has been unaffected by the scaling. In Table 4 the dependence of $N$ and of the relative accuracy of the numerical solution from $\alpha$ is shown; in (18) we set $E P S=10^{-7}$ only because we could not establish a more accurate result independently. We notice that the advantage obtained with the introduction of the scaling parameter can be considerable; for instance, in example (25) $N$, the number of terms required in summation (8), is only $30 \%$ of the corresponding value required to obtain the same accuracy without scaling.

Example 3. In the two following cases our method does not work well unless the appropriate scaling is used:

$$
\begin{aligned}
& f\left(x^{2}\right)=e^{0.8 x^{2}} \\
& f\left(x^{2}\right)=e^{0.85 x^{2}}
\end{aligned}
$$


TABLE 4

\begin{tabular}{|c|c|c|c|c|c|c|c|c|}
\hline & \multicolumn{2}{|c|}{ Example (25) } & \multicolumn{2}{c|}{ Example (26) } & \multicolumn{2}{c|}{ Example (27) } & \multicolumn{2}{c|}{ Example (28) } \\
\hline$\alpha$ & $N$ & $\begin{array}{c}\text { Relative } \\
\text { accuracy } \\
\text { of numerical } \\
\text { results }\end{array}$ & $N$ & $\begin{array}{c}\text { Relative } \\
\text { accuracy } \\
\text { of numerical } \\
\text { results }\end{array}$ & $N$ & $\begin{array}{c}\text { Relative } \\
\text { accuracy } \\
\text { of numerical } \\
\text { results }\end{array}$ & $N$ & $\begin{array}{c}\text { Relative } \\
\text { accuracy } \\
\text { of numerical } \\
\text { results }\end{array}$ \\
\hline 0.47 & 30 & $0.1 \cdot 10^{-8}$ & 52 & $0.8 \cdot 10^{-7}$ & 31 & $0.1 \cdot 10^{-7}$ & 40 & $0.1 \cdot 10^{-6}$ \\
0.67 & 22 & $0.4 \cdot 10^{-9}$ & 44 & $0.7 \cdot 10^{-9}$ & 24 & $0.2 \cdot 10^{-7}$ & 32 & $0.1 \cdot 10^{-6}$ \\
0.82 & 6 & $0.9 \cdot 10^{-14}$ & 37 & $0.3 \cdot 10^{-9}$ & 23 & $0.1 \cdot 10^{-7}$ & 24 & $0.1 \cdot 10^{-6}$ \\
0.88 & 16 & $0.6 \cdot 10^{-9}$ & 33 & $0.1 \cdot 10^{-6}$ & 25 & $0.1 \cdot 10^{-7}$ & 21 & $0.1 \cdot 10^{-6}$ \\
1.00 & 22 & $0.4 \cdot 10^{-8}$ & 28 & $0.3 \cdot 10^{-7}$ & 29 & $0.1 \cdot 10^{-7}$ & 24 & $0.1 \cdot 10^{-6}$ \\
1.11 & 27 & $0.4 \cdot 10^{-8}$ & 17 & $0.2 \cdot 10^{-7}$ & 33 & $0.1 \cdot 10^{-7}$ & 28 & $0.1 \cdot 10^{-6}$ \\
1.29 & 35 & $0.9 \cdot 10^{-8}$ & 22 & $0.1 \cdot 10^{-7}$ & 40 & $0.1 \cdot 10^{-8}$ & 35 & $0.1 \cdot 10^{-6}$ \\
1.49 & 44 & $0.1 \cdot 10^{-8}$ & 35 & $0.2 \cdot 10^{-7}$ & 47 & $0.5 \cdot 10^{-8}$ & 43 & $0.1 \cdot 10^{-6}$ \\
\hline
\end{tabular}

with $\omega=4$. Taking $E P S=10^{-12}$ in (18), with no scaling we could obtain for (29) only three significant correct decimal digits, since $\left|b_{0} V_{0}\right| \simeq 0.046,\left|b_{M} V_{M}\right| \simeq 0.40 \cdot 10^{5}$ with $M=17,\left|b_{M^{\prime}} V_{M^{\prime}}\right| \simeq 0.36 \cdot 10^{-10}$ with $M^{\prime}=60,\left|b_{N} V_{N}\right| \simeq 0.79 \cdot 10^{-21}$ with $N=77$. The case (30) is very intractable. With no scaling the method yields no meaningful result; more precisely, we have $\left|b_{0} V_{0}\right| \simeq 0.061,\left|b_{M} V_{M}\right| \simeq 0.36 \cdot 10^{8}$ with $M=23,\left|b_{M^{\prime}} V_{M^{\prime}}\right| \simeq 0.12 \cdot 10^{-7}$ with $M^{\prime}=74,\left|b_{N} V_{N}\right| \simeq 0.14 \cdot 10^{-19}$ with $N=94$ and $I^{(N)}(\omega)=-0.12 \cdots 10^{-7}$, while the exact solution is $I(\omega)=0.87 \cdots$ $10^{-11}$. In both cases (29) and (30) by using a scaling factor we can obtain 12 significant correct decimal digits; this happens when $\mid \alpha=1.87$ giving $\left|b_{0} V_{0}\right| \simeq$ $0.59 \cdot 10^{-6},\left|b_{M} V_{M}\right| \simeq 0.38 \cdot 10^{-4}$ with $M=7,\left|b_{M^{\prime}} V_{M^{\prime}}\right| \simeq 0.16 \cdot 10^{-19}$ with $M^{\prime}=$ $37,\left|b_{N} V_{N}\right| \simeq 0.65 \cdot 10^{-22}$ with $N=40$, for (29) and $\alpha=2.40$ giving $\left|b_{0} V_{0}\right| \simeq$ $0.59 \cdot 10^{-10},\left|b_{M} V_{M}\right| \simeq 0.49 \cdot 10^{-9}$ with $M=4,\left|b_{M^{\prime}} V_{M^{\prime}}\right| \simeq 0.16 \cdot 10^{-24}$ with $M^{\prime}=$ $30,\left|b_{N} V_{N}\right| \simeq 0.16 \cdot 10^{-24}$ with $N=30$ for (30).

The numerical examples in this section indicate that the method, used in conjunction with the scaling technique, has some generality and may even be used successfully in cases when the Laguerre expansion on which it is based diverges.

Unfortunately, we know of no prescription for choosing the scaling parameter $\alpha$, except by intelligently applied trial and error. This problem is similar to that of determining an optimum step size in numerical differentiation.

7. Concluding Remarks. In this paper we have described in some detail the application of the Bakhvalov and Vasil'eva method to evaluate numerically integrals of the form

$$
I(\omega)=\int_{0}^{\infty} e^{-x^{2}} J_{0}(\omega x) f\left(x^{2}\right) x d x
$$

for moderate and large values of $\omega$. The method is based on an expansion of $f\left(x^{2}\right)$ in terms of Laguerre polynomials. In cases when this expansion converges reasonably rapidly the method is easy to use and is effective. 
However, when this is not the case, the method is difficult to use and in unmodified form may produce meaningless results. In Sections 4-6 we have described a way of modifying the problem using a scaling parameter $\alpha$. This may be used either to reduce the number of terms required in the expansion thus reducing the cost, or to remove an unpleasant numerical characteristic which can occur and result in considerable loss of numerical significance. Unfortunately, we have no general prescription for choosing the scaling parameter.

However, we have shown that the scope of the unmodified method may be significantly widened using scaling.

Remark. The method expounded upon can be easily generalized to the evaluation of

$$
I_{\nu}(\omega)=\int_{0}^{\infty} e^{-x^{2}} J_{\nu}(\omega x) f\left(x^{2}\right) x^{\nu+1} d x, \quad \nu>-1
$$

Let

$$
f\left(x^{2}\right) \simeq \sum_{k=0}^{N} b_{k}^{(v)} L_{k}^{(\nu)}\left(x^{2}\right) .
$$

Using the procedure of Section 2 we find

$$
I_{\nu}(\omega) \simeq \sum_{k=0}^{N} b_{k}^{(\nu)} U_{k}
$$

with

$$
U_{k}=\frac{e^{-\omega^{2} / 4}}{\Gamma(\nu+k+1)}\left(\frac{\omega}{2}\right)^{2 k+\nu}
$$

and

$$
b_{k}^{(\nu)}=\int_{0}^{\infty} e^{-y} y^{\nu} L^{(\nu)}(y) f(y) d y .
$$

The computation of $b_{k}^{(\nu)}$ is performed in the standard way, and formulas for $U_{k}$ similar to (9)-(10) can be derived.

We remark that Linz's method [7] generalized to (23) applies only for integer $\nu$.

Acknowledgment. I am grateful to Dr. J. N. Lyness for many helpful discussions and constructive suggestions.

Istituto di Calcoli Numerici

Universita di Torino

Torino, Italy

1. N. S. BAKHVALOV \& L. G. VASIL'EVA, "Evaluation of the integrals of oscillating functions by interpolation at nodes of Gaussian quadratures," USSR Comp. Math. and Math. Phys., v. 8, 1968, pp. $241-249$.

2. J. BALAZS \& P. TURAN, "Notes on interpolation. IX," Acta Math. Acad. Sci. Hungar., v. 16, 1965, pp. 215-220.

3. W. BARRETT, “Convergence properties of Gaussian quadrature formulas," Comput. J., v. 3, 1960, pp. 272-277.

4. C. W. CLENSHAW \& A. R. CURTIS, "A method for numerical integration on an automatic computer," Numer. Math., v. 2, 1960, pp. 197-205.

5. R. J. GLAUBER, Lectures in Theoretical Physics, Vol. 1, Interscience, New York, 1959. 
6. I. S. GRADSHTEYN \& I. M. RYZHIK, Table of Integral Series and Products, Academic Press, New York, 1965.

7. P. LINZ, "A method for computing Bessel function integrals," Math. Comp., v. 26, 1972, pp. $509-513$.

8. I. M. LONGMANN, "Note on a method for computing infinite integrals of oscillatory functions," Proc. Cambridge Philos. Soc., v. 52, 1956, pp. 764-768.

9. J. N. LYNESS, "Adjusted forms of the Fourier coefficient asymptotic expansion and applications in numerical quadrature," Math. Comp., v. 25, 1971, pp. 86-104.

10. T. N. L. PATTERSON, "On high precision methods for the evaluation of Fourier integrals with finite and infinite limits," Numer. Math., v. 27, 1976, pp. 41-52.

11. R. PIESSENS \& A. HAEGEMANS, "Numerical calculation of Fourier transform integrals," Electron. Lett., v. 9, 1973, pp. 108-109.

12. N. M. STEEN, G. D. BYRNE \& E. M. GELBARD, "Gaussian quadratures for the integrals $\int_{0}^{\infty} \exp \left(-x^{2}\right) f(x) d x$ and $\int_{0}^{b} \exp \left(-x^{2}\right) f(x) d x$," Math. Comp., v. 23, 1969, pp. 661-671. 